\title{
SUR LES CYSTICERCOÏDES DE QUELQUES DIPYLIDIUM
}

\author{
Par C.-R. LOPEZ-NEYRA
}

Nos connaissances sur le cycle évolutif des Dipylidium sont assez restreintes : Melnikow (1869) signale dans la cavité générale du Trichodectes canis Ret $z$. le cysticercoïde du D. caninum L., 1767 : Les recherches de Villot (1883), Sonsino (1888), Grassi et Rovelli (1888 $a$ et $b, 1889)$ et surtout Joyeux $(1916,1920)$ ont établi, par l'expérimentation rigoureuse, le développement complet de $D$. caninum, en ce qui concerne son évolution chez les puces : Ctenocephalus canis (Curtis, 1826) et Pulex irritans I., 1758. Ces insectes s'infestent à l'état larvaire en absorbant les oufs du cestode, mais le cysticercoïde (Cryptocystis trichodectis Villot) ne se développe que lorsque la puce, après sa métamorphose, est sortie de son cocon.

Diamare (1892), comparant le scolex du cystique avec celui de la forme adulte, assimile le Cysticercus acanthotetra Parona, 1886, trouvé dans les parois intestinales de la couleuvre Zamenis viridiflavus Wagl., au D. trinchesei Railliet, 1893, parasite du chat, et le Cysticercus rostratus Mingazzini, 1893, de la même couleuvre au D. echinorhynchoides Sonsino, 1889 (Diamare, 1894), du Megalotis cerdo Skje. Ce D. echinorhynchoides a été transmis expérimentalement au chat, par Ch. Joyeux (1923).

Marchi a décrit (1872), dans les parois intestinales de Tarentola mauritanica $\mathrm{L}_{\text {., }}$ un cystique à quatre rangées de crochets (70) d'inégale longueur $(15-16 \mu$ à 5,8-6 $\mu)$, qu'il a essayé de faire évoluer chez divers animaux (1878) : un vautour fauve et un chat, sans succès; par contre, en le faisant ingérer à une chouette (Strix noctua Boie) et en sacrifiant cet oiseau au bout de quelques jours, il a. vu de jeunes ténias commençant à se développer dans l'intestin ; mais on ne connaît pas de Dipylidium chez la chouette. Ce Cysticercus ascalabotidis a été retrouvé par Rizzo (1902) chez Lacerta ayilis à Catane et, d'après Diamare (1894), par Mingazzini chez Seps chalcides Dum. et Bib.

Muñuz Medina et moi-mème (1919), ainsi que larrot et Joyeux (1920), avons établi expérimentalement la concordance de deux cysticercoïdes de Tarentola mauritanica L., avec des ténias du chat: l'un à quatre rangées de crochets, identique au $C$. acanthotelra Parona, du Zamenís viridiflabus, représente la forme larvaire de

Axiales de Parasitologie, T. V, No $3 .-1^{\text {er }}$ juillet $1927, p .245-218$. 
$D$. trinchesei; l'autre à 14-16 rangées de crochets en forme d'aiguillons de rosier et déjà signalé par Sonsino (1897) représente le cysticercoïde de $D$. chyzeri v. Ratz, 1897.

L'hôte intermédiaire normal de ces ténias est la Tarentola mauritanica L. en Espagne, au Maroc (C.-R. Lopez-Neyra), en Algérie et en Tunisie (Joyeux) et l'Hemidactylus turcicus L. à Athènes (Blanc et Caminopetros, 1921). Mais ces cystiques sont très ubiquistes et peuvent se développer chez un grand nombre de reptiles qui sont alors des hôtes intermédiaires occasionnels, n'étant jamais ou rarement la proie du chat: Zamenis viridiflavus Wagl., Z. hippocrepis L., Tropidonotus viperinus Latr., Cerastes cornutus L., rarement Gongylus ocellatus Gm., et Bufo mauritanicus Schl.

Nous avons essayé en vain de faire développer ces cysticercoïdes chez l'hôte intermédiaire. Les recherches de Joyeux (1923), qui a fait avaler des oufs mûrs à la tarente, ont été également sans succès, et cet auteur se demande s'il n'existerait pas deux hôtes intermédiaires; le premier étant peut-être un insecte coprophage qui serait avalé par le reptile.

Joyeux (1921) a décrit dans le mésentère d'une vipère à cornes (Cerastes cornutus L.) un cysticercoïde ayant la forme typique de ceux des Dipylidium qu'il a rapportés, soit à $D$. triseriale Lühe, 1896, soit à une espèce voisine inconnue ou mal décrite. En comparant cette larve avec les vers adultes, je crois qu'elle pourrait peut-être correspondre au D. zschokkei Hungerbühler, 1910, du Cynictis penicillata de l'Afrique du Sud, espèce possédant trois couronnes de crochets, quelques-uns téniiformes. Voici ses caractéristiyues :

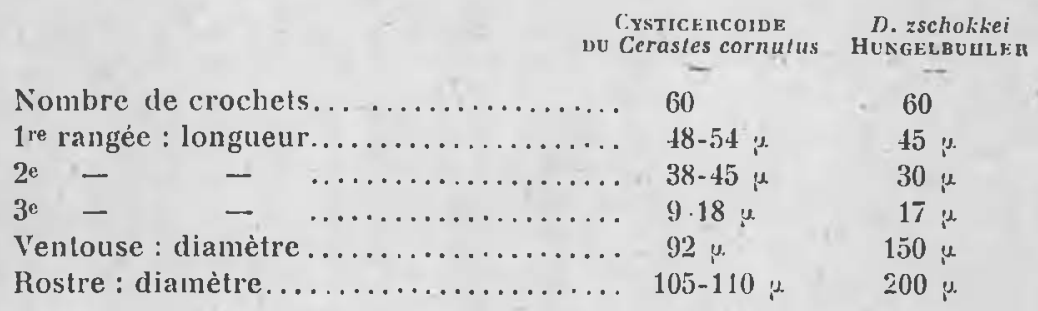

Par contre, Joyeux (1923) a pu trouver le cysticercoïde de D. triseriale Lühe, 1896, encapsulé dans le péritoine chez Chlorophis irreqularis Leach. récolté en Guinée française par le Dr Gendre. Le cystique ressemble tcut à fait à celui des Dipylidium. Le rostre porte a peu près soixante crochets disposés sur trois rangs; ils sont donc un pen plus grands pour les deux premières rangées que ceux 
mesurés par Lühe (1896) et Joyeux (1923), mais c'est là une différence insignifiante, et l'analogie entre les crochets de ce cystique et ceux du ver adulte est tellement évidente que Joyeux a pu affirmer le cycle évolutif, sans avoir recours à l'expérimentation.

En 1921, nous avons trouvé, avec la collaboration de MuñozMedina, un nouveau cestode parasite du chat domestique, le $D$. quinquecoronatum, espèce voisine du $D$. trinchesei, mais avec 5 couronnes de crochets mesurant comme longueur totale : 68-72 $\mu$, $56-60 \mu, 32-40 \mu, 22-29 \mu$ el $1016 \mu$. Nous avous ell aussi l'occassion de signaler l'existence de cysticercoïdes, encapsulés dans les parois intestinales, le foie et la région hilaire du poumon de Tarentola mauritanica L., à Grenade, semblables par leur morphologie et leur taille au cystique (Cysticercus acanthotetra Parona) étudié par Joyeux et Parrot et par nous, mais avec cinq couronnes de crochets mesurant $69,56,32,21$ et $10-12 \mu(1)$. Nous n'avons pu, faute de matériel, donner de preuve expérimentale.

Ayant pu disposer, pendant le mois d'octobre, d'une tarente parasitée par ces cysticercoïdes, j'ai essayé d'infester un jeune chat ( 20 jours) en faisant ingérer 6 cysticercoïdes à 5 couronnes de crochets ; un autre jeune chat a servi de témoin. Les animaux ont été sacrifiés au bout de 15 jours : chez le premier, j'ai trouvé, dans l'intestin, quatre Dipylidium avec les caractères du D. quinquecoronatum, montrant des organes sexuels bien formés dans leurs derniers anneaux, mais sans capsules utérines développées.

\section{RÉSUMÉ}

Tarentola mauritanica est l'hôte intermédiaire normal de $D$. chy$z c r i$, de $D$. trinchesei et de $D$. quinquecoronatum en Espagne. Pendant la saison chaude (août à octobre), on trouve les cysticercoïdes de $D$. chyzeri chez 25 p. 100 de ces reptiles, ceux de $D$. trinchesei chez 12 p. 100 et ceux de 1 . quinquecoronatum chez seulement 6 p. 100.

(1) Dislance de l'extrénité du manche à la pointe de la lame. 


\section{BibLIOGRAPHIE}

Blanc (G.) et Caminorèthos (J.). - Le cysticercoüde d'Ilenidaclylus turcicus. Bull. soc. path. exol. Paris, XIV, 1921, p. 20-21.

Diamahe (V.). - Il genere Dipylidium Leuckart. Alli $R$. Acad. sc. fis e nat. d Napoli (2), II, 1892.

Grassı (B.). - a) La puce dei cane (Pulex serraticeps) el' ordinario ospilo inlermediaro della T'. cucumerina. Not. prev. Catania, 1888.

Ghassi (B.) et Rovelu (G.). - b) Iutosuo allo sviluppo dei Cestodi. Rend. $k$ Accad. dei Lincei An 11.285 (4), fase. 12, 1888, p. 700-702.

- Embriologische Forschungen an Cestoden. Centralbl. f. Backt. u. Parasit, V, 1889 , p. 370-377 et $401-410$.

HсNGenнёнten, 1910. - Studien an Gyrocolyle und Cestoden. Jena. Denlischr. med. Ges.; XVI, 1910, p. 495-522, pl. XVIII-XIX.

Joreex (Ch.). - Sur le cycle évolutif de quelques cestodes. Bull, soc. palh, exol. Paris, IX, 1916, p. 578-583.

- Cycle évolutif de quelques cestodes. Recherches expérimentales. Supplém. II an Bull. biol. de France el de Belgique. Paris, 1920, 210 p., 7 pl.

- Description d'un cysticercoüde de Cerastes cornulus. Arch. Inst. Pasteur d'A frique du Nord, I, 1921, p. 383-387.

- Recherches sur la faune helmintologique africaine. Arch. Inst. Paslent de Tunis, XII, 192:3, p. 119-167.

Lopez-Neyia (C.-R.) et Musoz-Menixa (J.-M.). - Estudio del ciclo evolutivo seguido por algunas especies correspondientes al genero Dipylidium Leuckart. Bol. R. soc. esp, hist. nat. Madrid, XIX, 1919, p. 494-504.

- Dipylidium quinquecoronalum n. sp. pasasito intestinal del gato domestico. Bol. R. soc. esp, hist. nat. Madrid, XXI, 1921, p. 421-426.

Manchi (P.). - Sopra un nouvo Cestocle trovato nell' Ascalaboles maurilanicus. Alli soc. ilul. sc. nat., XV, 1872, p. 305-306; C. R. Ass. franc. p. l'av. d. sc. Paris, VII, 1872, p. 757.

Melnikov. - Ueber die Ingerzustände der $T$. cucumerina. Arch. $f$. Nalurgesch. Jahrg. 35, I, 1869, p. 62-70, pl. I.

Pанвот (I..) et Joyeux (Ch.). - Les eysticercoïdes de Tarentola muturitanica L. et les ténias du cluat. Bull. soc. palh. cxol. Paris, XIII, 1920, p. 687-695.

Rrzzo (A.). - La fauna elmintologica dei retilli nella provinvia di catania. Arch. Parasilologie, V'I, 1902, p. 26-40, fig. 1-12.

Sonsino ( $P^{2}$.); 1887. - Alcuni elminti racolti e esservato di recente in Pisa. Alli. soc. losc. d. sc. nat., $\mathrm{X}, 1887, \mathrm{p} .253-260$.

- Ricerche sugli ematozoi del cane e sul cyclo vitale della $T$. cucumerina. Alli soc. tosc. d. sc. mul., X, 1888, p. 1948, 2 pl.

Vılıot, 1883. - Mémoire sur les cysticerques des ténias. Ann. sc. nal. (6). Zool., $\mathrm{XV}, 1883 ; \mathrm{Rev} . \mathrm{sc}$. nal. (3), II, 1882.

Laboraloire de Zoologie el de Parasilologie. de la Fraculté de pharmacie de l'Lniversilé de Grenade. 